\title{
O uso da creatina no treinamento de força e na melhoria do desempenho físico
}

\author{
The use of creatine in strength training and improving physical performance \\ El uso de creatina en el entrenamiento de fuerza y la mejora del rendimiento físico
}

Recebido: 13/08/2021 | Revisado: 19/08/2021 | Aceito: 20/08/2021 | Publicado: 22/08/2021

\author{
João Pedro Cardozo dos Santos \\ ORCID: https://orcid.org/0000-0002-6284-6277 \\ Centro Universitário Fametro, Brasil \\ E-mail: joaopedrocardozo610@gmail.com \\ Gabriel Henrique da Silva Martins \\ ORCID: https://orcid.org/0000-0003-4792-5847 \\ Centro Universitário Fametro, Brasil \\ E-mail: martinsgabriel2242@gmail.com \\ José Carlos de Sales Ferreira \\ ORCID: https://orcid.org/0000-0002-1867-8229 \\ Centro Universitário Fametro, Brasil \\ E-mail: jcarlos.sales@gmail.com
}

\begin{abstract}
Resumo
Introdução: $\mathrm{O}$ treinamento resistido, também conhecido como treinamento de força, tornou-se uma das formas mais populares de exercício para melhorar a aptidão física e para condicionamento de atletas. A suplementação com creatina vem sendo a principal opção entre os atletas com características de força máxima, explosiva e velocidade. Objetivo Geral: Compreender efeitos da creatina em treinamentos de força e condicionamento físico por meio dos objetivos específicos da pesquisa que são: caracterizar a creatina e suas fontes naturais; enfatizar sobre a importância do nutricionista na prescrição de suplementos na prática de treinamento de força; apontar os efeitos da suplementação com creatina no treinamento de força e na melhoria do condicionamento físico. De fato, quando associado à suplementação de creatina, o treinamento de força ou resistido tem mostrado maior benefício para o aumento de massa corporal, força máxima, aumento da capacidade de elevação de peso, aumento da massa livre de gordura e aumento das proteínas miofibrilares. Metodologia: Trata-se de uma revisão bibliográfica com abordagem descritiva. Resultado e Discussão: Para análise dos dados foi utilizada abordagem qualitativa onde a aplicação desse método considera que há uma relação dinâmica entre o mundo real e o objeto de pesquisa, que não pode ser traduzido em números. Os dados selecionados foram apresentados em forma de texto descritivo, reunindo todos os autores selecionados acerca da temática de pesquisa. Conclusão: Através do desenvolvimento desse estudo, foi possível demonstrar através da literatura, os potenciais efeitos positivos da suplementação da creatina manifesta como um futuro e propício campo de estudo.
\end{abstract}

Palavras-chave: Creatina; Fontes naturais; Feitos da suplementação; Prescrição de suplementos.

\begin{abstract}
Introduction: Resistance training, also known as strength training, has become one of the most popular forms of exercise to improve the physical fitness and conditioning of athletes. Creatine supplementation has been the main choice among athletes with characteristics of maximum strength, explosiveness and speed. General Objective: Understand the effects of creatine in strength training and physical conditioning through the specific objectives of the research, which are: to characterize creatine and its natural sources; emphasize the importance of the nutritionist in prescribing supplements in the practice of strength training; to point out the effects of creatine supplementation on strength training and on improving physical fitness. In fact, when combined with creatine supplementation, strength or resistance training has the greatest benefit in increasing body mass, maximal strength, increasing weight lifting capacity, increasing fat-free mass, and increasing myofibrillar proteins. Methodology: This is a literature review with a descriptive approach. Results and Discussion: For data analysis, a qualitative approach was used, where the application of this method considers that there is a dynamic relationship between the real world and the research object, which cannot be translated into numbers. The selected data were selected in the form of descriptive text, bringing together all selected authors on the research topic. Conclusion: Through the development of this study, it was possible to demonstrate through the literature, the possible positive effects of creatine supplementation manifested as a future and favorable field of study.
\end{abstract}

Keywords: Creatine; Natural sources; Supplementation effects; Prescription supplements. 


\begin{abstract}
Resumen
Introducción: El entrenamiento de resistencia, también conocido como entrenamiento de fuerza, se ha convertido en una de las formas de ejercicio más populares para mejorar la condición física y el acondicionamiento de los deportistas. La suplementación con creatina ha sido la principal elección entre los deportistas con características de máxima fuerza, explosividad y velocidad. Objetivo general: Comprender los efectos de la creatina en el entrenamiento de fuerza y acondicionamiento físico a través de los objetivos específicos de la investigación, que son: caracterizar la creatina y sus fuentes naturales; enfatizar la importancia del nutricionista en la prescripción de suplementos en la práctica del entrenamiento de fuerza; señalar los efectos de la suplementación con creatina en el entrenamiento de fuerza y en la mejora de la condición física. De hecho, cuando se combina con suplementos de creatina, el entrenamiento de fuerza o resistencia tiene el mayor beneficio para aumentar la masa corporal, la fuerza máxima, aumentar la capacidad de levantamiento de pesas, aumentar la masa libre de grasa y aumentar las proteínas miofibrilares. Metodología: Se trata de una revisión de la literatura con enfoque descriptivo. Resultados y Discusión: Para el análisis de datos se utilizó un enfoque cualitativo, donde la aplicación de este método considera que existe una relación dinámica entre el mundo real y el objeto de investigación, que no se puede traducir en números. Los datos seleccionados fueron seleccionados en forma de texto descriptivo, reuniendo a todos los autores seleccionados sobre el tema de investigación. Conclusión: A través del desarrollo de este estudio, fue posible demostrar a través de la literatura, los posibles efectos positivos de la suplementación con creatina manifestados como un campo de estudio futuro y favorable.
\end{abstract}

Palabras clave: Creatina; Fuentes naturales; Hazañas de suplementación; Prescripción de suplementos.

\title{
1. Introdução
}

O treinamento resistido, também conhecido como treinamento de força ou com pesos, tornou-se uma das formas mais populares de exercício para melhorar a aptidão física e para condicionamento de atletas. É caracterizado pelo exercício que exige que a musculatura corporal se movimente ou tente se movimentar contra uma força oposta, geralmente exercida por algum tipo de equipamento como: pesos, tiras elásticas, pliométricos e de corrida em ladeiras (Fleck \& Kraemer, 2017).

Nesse contexto, a suplementação com creatina vem sendo a principal opção entre os atletas com características de força máxima, explosiva e velocidade; tornando comum entre atletas da luta, ciclistas, nadadores, fisiculturistas e principalmente entre os frequentadores de academia (Stábile et al., 2017).

Contudo, a suplementação com creatina vem sendo utilizada sem nenhum conhecimento ou orientação de um profissional de nutrição. Muitas vezes utilizada por indicação de profissionais que não são competentes da área de nutrição ou ainda por pessoas próximas ao usuário que deseja utilizar algum suplemento alimentar, resultando em um desempenho negativo nos resultados obtidos no treinamento de força ou condição física.

Desse modo, a pesquisa busca responder a seguinte problemática: quais benefícios e malefícios para pessoa que utilizam a creatina no treinamento de força e na melhoria do condicionamento físico? Grande parte dos atletas e praticantes de musculação usam a suplementação com intuito principal de aumentar a massa muscular através da força, velocidade e potência nos exercícios, levando ganhos significativos na melhoria da performance física. No entanto, sem indicação ou acompanhamento do profissional de nutrição pode não ser tão eficiente no organismo ou ainda causar efeitos colaterais indesejados.

O interesse pelo tema surgiu da necessidade de disseminar sobre o uso da creatina entre os frequentadores de academia e atletas. Devido ao aumento da prática de atividade física as pessoas buscam recursos com a finalidade de atingir padrões estéticos almejados. Desse modo, há uma grande busca pelo suplemento por meio da creatina que proporciona melhoras de desempenho físico e condição física.

Contudo, a oferta equilibrada de nutrientes advindas da suplementação alimentar é a base para um melhor desempenho físico, prevenção de fadiga muscular e ainda a redução de lesões, uma vez que gera o combustível par ao trabalho biológico e as substâncias químicas para extrair e utilizar a energia proveniente dos alimentos (Galvão et al., 2017). 


\section{Metodologia}

O objetivo da pesquisa foi de caráter descritivo, propondo a identificação, registro e análise das características, fatores ou variáveis que se relacionam com o fenômeno ou processo (Perovano, 2014). Além disso, busca-se destacar os benefícios e malefícios do uso da creatina no treinamento de força e na melhoria do condicionamento físico registrados na literatura nacional.

Quanto ao procedimento, a pesquisa foi bibliográfica onde é desenvolvida a partir de material publicado, constituído principalmente de livros e artigos em periódicos, fazendo parte também da revisão da literatura as monografias, dissertações e teses (Lakatos \& Marconi, 2010).

A coleta de dados foi totalmente eletrônica por meio da internet, no qual será norteada pelas palavras chaves envolvidas na temática estudada como: uso da creatina no treinamento de força; suplementação e treinamento; efeitos da creatina no treinamento de força e condicionamento físico; características da creatina.

Pesquisado por meio do banco de dados da REDALYC, LYLACS, MEDLINE, SCIELO e BVS, publicações a partir de 2011, e livros disponibilizados no Google Books, onde todo material pesquisado será analisado e revisados e contará com visões e opiniões de especialistas da área, também foram analisados alguns livros envolvendo a temática abordada.

Para análise dos dados foi utilizada abordagem qualitativa onde a aplicação desse método considera que há uma relação dinâmica entre o mundo real e o objeto de pesquisa, que não pode ser traduzido em números.

A partir da leitura bibliográfica e seletiva, foi selecionado todo conteúdo que norteará a temática abordada para o desenvolvimento teórico da pesquisa. Utilizando a técnica de fichamento, toda teoria e autores selecionados foram elencados objetivando melhor compreensão e análise da temática em questão.

Os critérios de inclusão utilizados serão: publicações a partir de 2011; publicações em Língua Portuguesa; publicações da REDALYC, SCIELO e BVS; somente livros e periódicos. Os critérios de exclusão utilizados serão: publicações menos de 10 anos; publicações em língua estrangeiras; materiais como TCC; dissertações, teses, trabalhos.

Os dados selecionados foram apresentados em forma de texto descritivo, reunindo todos os autores selecionados acerca da temática de pesquisa

\section{Resultados e Discussão}

\subsection{Treinamento de força e condicionamento físico}

Atividade física refere-se a qualquer movimento do corpo que envolva esforço, e, portanto, exige energia além daquela necessária em repouso. Já exercício físico é uma forma mais direcionada, ou específica, de atividade física para melhorar da saúde. Tanto a atividade física como o exercício incluem movimentos que exigem energia, mas a finalidade do exercício é melhorar ou manter o condicionamento físico (brushman, 2017).

Para Santarém (2015, p. 5) a atividade física:

Corresponde a contração muscular esquelética ocorrendo o consumo de energia e estímulos de diversas funções orgânicas. A contração muscular geralmente leva ao movimento do corpo, mas nem sempre. Quando músculos que realizam movimentos opostos nas articulações são ativados simultaneamente, não ocorre movimento, sendo bastante comum nas atividades diárias, no trabalho e no esporte.

Nesse contexto, a contração muscular pode ocorrer de três formas básicas: a contração concêntrica que ocorre quando o músculo se encurta; contração excêntrica é a que ocorre quando o músculo se alonga; e a contração estática ou isométrica é a que ocorre quando a força produzida não consegue vencer a resistência e não ocorre o movimento (Chandler \& Brown, 2015). 
A força do ponto de vista da física, é representada pela expressão do produto da massa pela aceleração, porém, quando o assunto é execução de movimentos e exercícios, a força fica representada pela superação de uma dada resistência que vem por meio da contração muscular (Uchida et al., 2018).

$\mathrm{O}$ treinamento de força sempre foi associado ao aumento da massa muscular, sendo um meio para atingir uma hipertrofia muscular e o aumento da força máxima, importante para muitos esportes (Bean, 2016).

Para Fleck e Kraemer (2017, p. 1):

Os termos treinamento de força, treinamento com pesos e treinamento resistido são todos utilizados para descrever um tipo de exercício que exige que a musculatura corporal se movimente ou tente se movimentar contra uma força oposta, geralmente exercida por algum tipo de equipamento.

Em relação aos praticantes de atividade física, é oportuno definir o Treinamento de força ou resistido, é uma educação para suportar exercícios sistemáticos submetidos a um esforço contrário e é um método essencial nos programas de treinamento para indivíduos com os mais diversos objetivos e níveis de aptidão física (Zanelli et al., 2015).

Define Sharkey (2015, p. 22) que:

A aptidão física é a capacidade de realizar esforços. Essa condição é determinada pelo grau de aprimoramento das diversas qualidades de aptidão física. Contudo, o termo aptidão físico engloba duas categorias principais: a capacidade aeróbica que se refere à capacidade do indivíduo para realizar tarefas continuas, tais como caminhar, correr, pedalar, esquiar, remar e outras que envolvem grandes músculos; e a capacidade muscular inclui: força, resistência e flexibilidade, bem como potência.

Desse modo, o entusiasmo da aptidão física, o praticante amador de musculação e o atleta esperam ganhos em força ou tamanho muscular (hipertrofia) a partir de um programa de treinamento de força. Várias modalidades desse treinamento como isocinético, resistência variável, isométrico e pliométricos podem ser utilizadas para atingir tais objetivos (Uchida et al., 2018).

Sendo esse tipo de treinamento uma das formas mais populares de exercício para melhorar a aptidão física dos atletas, o condicionamento físico e qualidade de vida. Nas modalidades desportivas, o treinamento de força torna-se de fundamental importância para o treinamento do atleta, onde a força manifesta-se na forma de resistência de força, força rápida, potência muscular e força máxima (Souza, 2015).

Nesse contexto, um programa de treinamento de força é um composto de diversas variáveis que interagem umas com as outras para oferecer um estímulo à

adaptação (Stábile et al., 2017).

O sucesso de qualquer programa de treinamento na produção de adaptações específicas depende da efetividade do estímulo de treinamento produzido por esse programa. O resultado de um bom programa de treinamento pode produzir alterações na composição corporal, na força, na potência, na hipertrofia muscular e no desempenho motor das pessoas (Fleck \& Kraemer, 2017).

\subsection{A creatina e suas características}

A creatina (ácido metil guanidino acético) é um aminoácido que, além de ser encontrado em alguns alimentos, também é produzido endogenamente, por um processo que envolve órgãos como fígado, rins e pâncreas e usa como substratos outros aminoácidos (glicina, metionina e arginina) (Amaral \& Nascimento, 2020).

A combinação polipeptídica entre a glicina, arginina e metionina tem como resultado em seu metabolismo final a produção de creatina, conhecida também como ácido metilguanidina-acético. Sua síntese acontece em duas etapas: a primeira, 
no rim, no qual, a glicina e a arginina são transformadas com base na enzima transaminidase, dessa forma a creatina circula até o fígado para receber um grupo metil, oriundo do aminoácido metionina (Menezes, Moraes \& Linhares, 2020).

Pode ser encontrado em alimentos como peixes e carnes, podendo ser sintetizado endogenamente no fígado, rins e pâncreas a partir de outros aminoácidos como a glicina, arginina e metionina. Está concentrado em sua maioria no músculo esquelético, sob a forma de fosfocreatina (Williams, Kreider \& Branch, 2017).

A fosfocreatina é a primeira reserva energética degradada durante atividades de alta demanda energética, que variam de dez segundos a cerca de um minuto, porem seus estoques são ressintetizados em poucos minutos, o que a torna importante em exercícios intermitentes (Prestes et al., 2016).

Segundo Lorenzeti, Júnior e Zanuto (2019, p. 72) a creatina pode ser encontrada no mercado em três modos:

Creatina monoidratada é um pó branco, fino, que se mistura facilmente em água; creatina micronizada referente so processo de micronização da creatina monoidratada, no qual o diâmetro das partículas de creatina é reduzido, melhorando sua absorção e sendo mais bem aproveitada pelo organismo; e a liofilização que é um processo de congelamento de produtos alimentícios que, posteriormente, passa para a produção do produto a vácuo, ocorrendo um aumento gradativo da temperatura, desidratando e retirando todo seu conteúdo de água sem alterar sua constituição química.

Existem diferentes protocolos para o uso da creatina, porém o mais comum é o método de saturação (sobrecarga). A captação de creatina pelas células musculares é um processo que ocorre de forma ativa contra o gradiente de concentração (transportador sódio-dependente), possivelmente envolvendo sítios específicos da membrana que reconhecem parte da molécula da creatina (Menezes, Moraes \& Linhares, 2020).

Na suplementação de atletas e praticantes de atividade física regular, o suplemento de creatina pode ser consumido na forma de pó ou cápsula. Ele só pode ser ingerido com a orientação de um médico ou nutricionista e o consumo deve ser realizado de acordo com a recomendação do profissional (Rodrigues, 2015).

Segundo Rodrigues (2015, p. 62) em relação ao uso da creatina no treinamento de força, recomenda-se que:

A suplementação com creatina não ultrapasse um período de 90 dias. Após 90 dias, a orientação é realizar uma pausa de pelo menos um mês pata evitar que o organismo cesse a produção da substância. A quantidade recomendada de creatina varia de acordo com o estado de saúde de cada indivíduo. Geralmente no caso de atletas a orientação varia entre 2 e 3 gramas ao dia.

No entanto, estudos indicam que a ingestão de $20 \mathrm{~g} / \mathrm{dia}(0,3 \mathrm{~g} / \mathrm{Kg}$ de peso) durante cinco dias é suficiente par aumentar os níveis de creatina muscular. Essa forma de suplementação é denominada de fase de sobrecarga. Posteriormente, desenvolvese a fase de manutenção, determinada pela ingestão de 2 a 5 gramas por dia $(0,03 \mathrm{~g} / \mathrm{kg}$ de peso) e que promove saturação dos músculos (Lorenzeti, Júnior \& Zanuto, 2019)

\subsection{Nutricionistas e a prescrição de suplementos}

A nutrição está intimamente relacionada com a atividade física. E por meio desta, pode-se melhorar a capacidade de rendimento do organismo, através de uma alimentação saudável e ingestão adequada de nutrientes (Matins \& Maia, 2018).

Os suplementos nutricionais são alimentos que servem para complementar com calorias e ou nutrientes a dieta diária de uma pessoa saudável, nos casos em que sua ingestão, a partir da alimentação, seja insuficiente, ou quando a dieta requer suplementação (Frade et al., 2016).

De acordo com a Resolução CFN No 390/2006: 
Suplementos nutricionais são produtos atribuídos à complementação da dieta, formulados de proteínas e aminoácidos, lipídios e ácidos graxos, carboidratos e fibras, vitaminas e minerais, podendo ser isolados ou unidos entre si (Conselho Federal de Nutricionista, 2006).

Um suplemento nutricional é o produto constituído de pelo menos um desses ingredientes: vitaminas; minerais; ervas e botânicos; aminoácidos; metabólitos; extratos ou combinações dos ingredientes acima. Estes podem ser classificados em hidroeletrolíticos, energéticos, proteicos, hipercalóricos, substituição parcial de refeições, creatinas e cafeínas. Podem ser encontrados em diversas apresentações como comprimidos, cápsulas, líquidos ou pó (Karkle, 2015).

O uso de suplementos alimentares desempenha um papel importante entre os praticantes de atividade física, principalmente os atletas, no sentido de manter a saúde e a forma física, aumentar a força e a massa muscular e melhorar a performance (Dos Santos \& Pereira, 2017).

Comentam Chandler e Brown (2015, p. 431) que o uso da creatina em treinamentos de força e melhorado condicionamento físico requer algumas precauções como:

Ingerir de 20 a $30 \mathrm{~g}$ nos primeiros 5-7 dias e depois ingerir 5g/dia, após, ingerir apenas 3-5g diariamente; estabelecer ciclos de uso (4-8 semanas) e não uso (4 semanas); comprar produtos de marca que tenha boa reputação e certificado de análise incluindo as seguintes informações: aparência, composição, teor de umidade, resíduos de ignição, contaminação microbial/patogênica; fermentos e bolores, venenos/metais pesados e outros contaminantes.

Sendo assim, o nutricionista é considerado o profissional capacitado e habilitado para orientar tanto o consumo de suplementos e fitoterápicos quanto uma dieta apropriada e específica para cada tipo de esporte praticado, dentro de academias e clubes com a finalidade de obter melhores resultados além de promover melhores escolhas alimentares levando assim a uma melhora na qualidade de vida (Frade et al., 2016).

Comentam Martins e Maia (2018, p. 726) que:

O profissional habilitado para orientar quanto à nutrição de indivíduos ou coletividade é o nutricionista, sendo assim, cabe a ele a produção do conhecimento sobre alimentação e a nutrição nas diversas áreas de atuação profissional, inclusive na nutrição para praticantes de exercícios físicos em diversos lugares. O mesmo deverá respeitar a legislação e ter competência para avaliar, diagnosticar e acompanhar o estado nutricional do paciente e planejar, prescrever, analisar, supervisionar e avaliar dietas e suplementos dietéticos para indivíduos sadios e enfermos.

Cabe ao nutricionista, profissional da área da saúde que possui área específica de atuação e atribuições, e por legislação definida, a prescrição de suplementos alimentares. O nutricionista deverá respeitar a legislação e ter competência para avaliar, diagnosticar e acompanhar o estado nutricional do paciente e planejar, prescrever, analisar, supervisionar e avaliar dietas e suplementos dietéticos para indivíduos sadios e enfermos (Karkle, 2015).

Segundo Brasil (2020, p. 140) para utilização do suplemento alimentar:

É preciso a leitura do rótulo dos produtos pelo Nutricionista é prática indispensável, já que esses produtos são utilizados como complemento aos alimentos já prescritos. Para que o uso dos suplementos proteicos seja benéfico ao usuário, a veracidade do rótulo deve ser avaliada.

Os rótulos deveriam descrever e informar os ingredientes dos suplementos, as propriedades nutricionais dos alimentos, dos produtos alimentares e dos suplementos, contribuindo tanto para a prescrição quanto para o consumo adequado destes produtos (Brasil, 2014).

Desse modo, o uso de suplementos nutricionais é considerado estratégia nutricional para indivíduos que não conseguem suprir as suas necessidades energéticas, portanto, é de competência do nutricionista auxiliar e informar os 
desportistas e praticantes de atividades físicas quanto à dieta, bem como a prescrição de suplementos nutricionais para a aquisição de ótimo estado nutricional (Moretti et al., 2018).

Suplementos liberados para a comercialização no Brasil podem conter componentes não aprovados e proibidos como agentes anabólicos, hormônios, beta- 2-agonista, estimulantes e diuréticos que são considerados como doping pela Agência Mundial Antidoping (Conselho Federal de Medicina, 2018. Wada, 2015).

\subsection{Efeitos da suplementação com creatina}

O monoidratado de creatina é muito usado por atletas de força e resistência par atingir o desempenho máximo. Afirma-se que pode sustentar a produção máxima de força por mais tempo que, no final, significa breves períodos de grandes atividades para atletas e esportistas e músculos maiores e mais fortes para atletas de peso (Bean, 2016).

Quando associado à suplementação de creatina, o treinamento de força ou resistido tem mostrado maior benefício para o aumento de massa corporal, força máxima, aumento da capacidade de elevação de peso, aumento da massa livre de gordura e aumento das proteínas miofibrilares.

Segundo Chandler e Brown (2015, p. 431) em relação aos benefícios da creatina incluem: "aumento da força dinâmica e potência em aproximadamente de 5 a $15 \%$; aumento do peso corporal e massa corporal magra em aproximadamente de $2 \%$ a 5\%; aumento no rendimento de sprints em aproximadamente de 1 a $5 \%$ ".

Há diversos benefícios do treinamento de força em relação a aptidão física e à qualidade de vida (Figura 1). No entanto, para que esses benefícios sejam otimizados, é preciso que a prescrição de exercícios seja específica para as características do indivíduo, o que significa considerar fatores como idade, aptidão física, histórico de treinamento e tolerância psicológica e física (Prestes et al., 2016). 
Figura 1. Benefícios do treinamento de força.

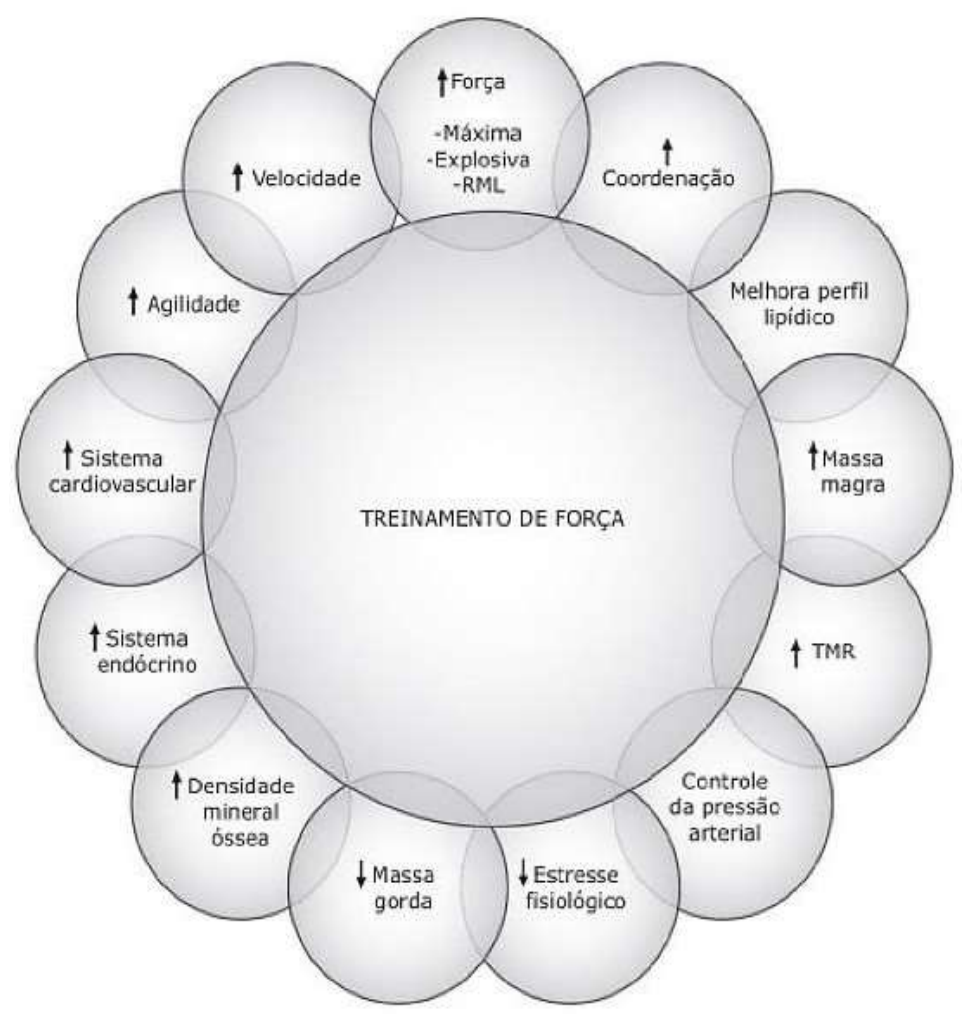

Fonte: Prestes et al. (2016).

Alguns desses benefícios são documentados na literatura onde destacam-se: o tratamento de doenças cardiovasculares, câncer, artrites, diabetes, obesidade, alémdos benefícios físicos como: aumento da força máxima, da potência e da resistência muscular, da coordenação motora, da velocidade, da agilidade, do equilíbrio e a prevenção e lesões (Bean, 2016).

Contudo, alguns estudos mostram que esta suplementação pode ser interessante para outas questões, entre elas a preservar a massa muscular em idosose prevenir doenças como Parkinson, Huntington e Alzheimer (rodrigues, 2015).

No geral, a suplementação de creatina a curto prazo pode contribuir para a massa corporal total aumentada, pelo menos em homens, ainda que muito desse aumento possa ser atribuído à retenção de água mais que à proteína contrátil aumentada.

Combinadas ao exercício físico resistido, a suplementação de creatina aumenta as concentrações de creatina total muscular (livre e fosforilada) em cerca de 25 a 37\%.Seu efeito no desempenho físico e na massa corporal magra é mais evidente em vegetarianos do que em não vegetarianos, isso ocorre pela falta de ingestão de carne(Lorenzeti, Júnior \& Zanuto, 2019).

Quando a creatina é ingerida, seus níveis séricos tipicamente aumentam por várias horas. O armazenamento de creatina no músculo ocorre essencialmentedurante os primeiros dias de suplementação. Depois, o excesso de creatina ingerido é excretado principalmente como creatina pela urina sendo clinicamente utilizado como indicador básico da degradação tecidual e/ou estresse renal, com uma pequenaquantidade sendo convertida para creatinina no músculo (Williams, Kreider \& Branch, 2017).

No entanto, evidências recentes indicam que a suplementação com creatina pode ser útil no tratamento de doenças, principalmente naquelas que resultam em atrofia e fadiga muscular. Também é utilizado no tratamento de pacientes com DPOC devido a redistribuição das fibras musculares predominantemente as do tipo II que apresentam maior capacidade anaeróbica e consomem mais fosfocreatina nos exercícios (Gualano, 2015).

Afirma (p. 64) que quanto a algum efeito colateral da creatina: 
Existe a possibilidade de o consumo do suplemento de creatina causar alguns efeitos colaterais. Um deles é a retenção de líquidos. A creatina é osmoticamente ativa, provoca aumento de seu conteúdo intracelular na forma de creatina livre e creatina fosfato no músculo, isso pode induzir um influxo de água para dentro da célula muscular, aumentando a água intracelular, o que pode dar uma falsa sensação de ganho de massa muscular.

Quando ingerido em altas doses superiores a 10 gramas, em cada dose pode levar a uma série de complicações como: dores no estômago, náuseas, diarreia e problemas cardíacos. Por virar creatinina nos rins pode levar a toxidade e insuficiência renal, uma vez que a filtração ficará prejudicada (Rodrigues, 2015).

\section{Conclusão}

A creatina desempenha papel fundamental no meio esportivo e tem se tornado um dos suplementos mais procurados por praticantes de atividade física. Boa parte dessa imensa procura e interesse pelo o suplemento é a comprovação da sua eficácia em muitos estudos apresentados, já que é um dos suplementos mais estudados atualmente. É importante destacar que apesar de toda a comprovação da sua capacidade, a creatina ainda é alvo de vários questionamentos, principalmente a respeito dos possíveis efeitos colaterais.

Vale enfatizar que de todos os estudos apresentados, nenhum se destacou por apresentar possíveis efeitos e malefícios que de alguma forma possa prejudicar o usuário. Necessário ressaltar sobre o "Ciclo de Saturação” onde alguns estudos destacaram que ao ser administrado doses de $0,3 \mathrm{~g} / \mathrm{kg} /$ dia durante $5-7$ dias e seguido de uma dose de manutenção variando de 3-5g/dia durante 3-4 semanas, mostrou-se eficiente e com resultados positivos na performance dos atletas apresentados. Porém é sempre importante destacar a individualidade de cada pessoa, e que a intensidade das atividades, estilo e hábitos devem ser considerados.

Por fim, com base nos estudos citados no presente trabalho pode-se concluir que parece não existir risco ou maléfico a saúde quando a suplementação de creatina é feita da maneira bem administrada, e que este suplemento continua se destacando por ser responsável por aumentar de forma significativa à força muscular e a hipertrofia em praticantes de musculação.

\section{Referências}

Amaral, A. de S., \& Nascimento, O. V. Efeitos da suplementação de creatina sobre o desempenho humano: uma revisão de literatura.

Azevedo, F. R. S. (2018) Efeitos da suplementação de creatina na performance de corredores velocistas: revisão de literatura.

Bean, Anita. (2016). O guia completo de treinamento de força. (5a ed.), Manole, 113.

BIUS - Boletim Informativo Unimotrisaúde em Sociogerontologia. (2020). 21(15), 1-20. https://periodicos.ufam.edu.br/index.php/BIUS/article/ view/8023.

Bouzas, J. C. M., Leite, M. S. R., Silva, F. M., \& Sousa, S.C. (2014). Creatina: estratégia ergogênica no meio esportivo: uma breve revisão. Rev. de Atenção à Saúde, 13(43), 52-60.

Brasil, C. C. B. (2020). Nutrição, análise e controle de qualidade de alimentos. Atenas.

Brasil. (2014). Ministério da Saúde. Guia alimentar para a população brasileira. MS.

Brushman, Barbara. (2017). Manual completo de condicionamento físico e saúde do ACSM. Phorte.

Cardoso, C. K de S., Oliveira, L. M., \& Azevedo, M. de O. (2017). Efeitos da suplementação de creatina sobre a composição corporal de praticantes de exercícios físicos. Revista Brasileira de Nutrição Esportiva, 11(61), 10-15.

Chandler, T., \& Jeff, B. L.E. (2015). Treinamento de força para desempenho humano. rtmed, 431.

Confortin, F. G., de Sá, C. A., \& Wildner, P. P. (2016). Avaliação da creatina associada à dextrose como suplemento nutricional ergogênico sobre a performance de atletas de futebol. RBNE-Revista Brasileira de Nutrição Esportiva, 10(56), 136-144.

Conselho Federal De Medicina. (2018). Medicamentos e suplementos nos exercícios e esportes. 72.

Conselho Federal De Nutricionista. (2006). Resolução CFN nº 390/2006: Regulamenta a prescrição dietética de suplementos nutricionais pelo nutricionista e dá outras providências. 
Da Silva, R. S. N., \& Toigo, A. M. (2016). Os efeitos do uso concomitante de cafeína e creatina nos exercícios físicos. Revista de Atenção à Saúde (antiga Rev. Bras. Ciênc. Saúde), 14(47), 89-98.

Dos Santos, E. A., \& Pereira, F. B. (2017). Conhecimento sobre suplementos alimentares entre praticantes de exercício físico. RBNE-Revista Brasileira de Nutrição Esportiva, 11(62), 134-140.

Faria, D. P. B. de. (2018). Suplementação de creatina no ganho de força e hipertrofia muscular em praticantes de treinamento de força: uma breve revisão narrativa. EAS, Revista Eletrônica Acervo Saúde, Sup. 13, S1476-S1483.

Fleck, J. S., \& kraemer, W. J. (2017). Fundamentos do treinamento de força muscular. (4a ed.), Artmed, 1.

Fontes, W., \& Moura, P. C. (2017). Percepção de eficiência da creatina enquanto suplemento ergogênico noorganismo de praticante de exercício resistido de força. Revista Brasileira de Ciências da Vida, 5(4), 19-19.

Frade, R. E., et al. (2016). Avaliação do consumo de suplementos nutricionais por frequentadores de uma academia da cidade de São Paulo. RBNE - Revista Brasileira de Nutrição Esportiva, 10(55), 50-58.

Francatto, E. C., Reggiolli, M. R., Maldonado, R. R., \& de Oliveira, D. S. (2016). A utilização de creatina por praticantes de musculação em academias na cidade de Mogi Mirim SP. Ciência \& Inovação.

Galvão, Francisca Gracielly Reinaldo et al. (2017). Importância do Nutricionista na Prescrição de Suplementos na Prática de Atividade Física: Revisão Sistemática. Revista e-ciência, 5(1), 52-59. http://www.revistafjn.com.br/revista/index.php/eciencia/article/view/245/245.

Gil, A. C. (2012). Métodos e técnicas de pesquisa social. (6a ed.), Atlas.

Gualano, Bruno. (2015). Suplementação com creatina: efeitos ergogênicos, terapêuticos e adversos. Manole, 113.

Karkle, Maiara Berndt. (2015). Uso de suplemento alimentar por praticantes de musculação e sua visão sobre o profissional nutricionista na área de nutrição. Revista Brasileira de Nutrição Esportiva, 9(53), 447-453, https://dialnet.unirioja.es/servlet/articulo?codigo=5296040.

Lakatos, Eva Maria, \& Marconi, Maria A. (2010). Fundamentos de metodologia científica: (7a ed.), Atlas.

Leite, M. M., de Oliveira Silva, A., Vieira, V. B., Funghetto, S. S., \& de Farias, D. L. (2018). Treinamento de força excêntrico e a produção de marcadores de dano muscular e marcadores inflamatórios em mulheres obesas. Programa de Iniciação Científica-PIC/UniCEUB-Relatórios de Pesquisa.

Leite, M. S. R., Sousa, S. C., Silva, F. M., \& Bouzas, J. C. M. (2015). Creatina: Estratégia ergogênica no meio esportivo. Uma breve revisão. Revista de Atenção à Saúde (antiga Rev. Bras. Ciênc. Saúde).

Lorenzeti, F. M., Júnior, L. C. C., \& Zanuto, R. (2019). Nutrição e suplementação esportiva: Aspectos metabólicos, fitoterápicos e da nutrigenômica. São Paulo: Phorte.

Macedo, T. A., de Oliveira, D. V., Domingues, W. J. R., \& Martineli, T. A. P. (2019). Efeitos de um programa de exercícios resistidos na força muscular de idosos. Renef, 8(11), 37-47.

Martins, A. D. B., \& Maia, J. K. S. (2018). Avaliação do conhecimento e prescrição de suplementação esportiva por profissionais de educação física e seus efeitos sobre alunos de academias. Revista Saúde em Foco, 10, 725-738.

Materko, W., \& Hallehandre, L. (2018). Efeito placebo no desempenho da força muscular em homens experientes em treinamento de força. RBNE-Revista Brasileira de Nutrição Esportiva, 12 (71), 374-379.

Menezes, C. dos S., Moraes, B. A., \& Linhares, P. S. D. (2020). Creatina: efeitos da suplementação. Referências em Saúde da Faculdade Estácio de Sá de Goiás-RRS-FESGO, 3(2). http://revistaadmmade.estacio.br/index.php/rrsfesgo/article/view/9197.

Moretti, S. M. B., et al. (2018). Perfil e consumo de suplementos nutricionais de praticantes de exercícios físicos em academias. RBNE-Revista Brasileira De Nutrição Esportiva, 12(74), 825-833. http://www.rbne.com.br/index.php/rbne/article/view/1146/821.

Neto, J. M. F. A., de Almeida, J. P. E., \& de Campos, M. F. (2017). Análise de marcadores celulares e bioquímicos sanguíneos para determinação de parâmetros de monitoramento do treinamento de praticantes de musculação. RBPFEX-Revista Brasileira de Prescrição e Fisiologia do Exercício, 11(70), 778783.

Panta, R., \& Silva Filho, J. N. (2015). Efeitos da suplementação de creatina na força muscular de praticantes de musculação: uma revisão sistemática. Revista Brasileira de Nutrição Esportiva, 9(54). 518-524.

Pazzetto, N., Silva, K. A., Lopes, J., \& Codonhato, R. (2018). Suplementação de creatina e treinamento resistido sobre a composição corporal de idosos: uma revisão sistemática. Caderno de educação física e esporte. Physical Education and Sport Journal, 16(1) 247-257.

Perovano, D. G. (2014). Manual de Metodologia Científica. Editora Juruá.

Prestes, J., Foschini, D., Marchetti, P., Charro, M., \& Tibana, R. (2016). Prescrição e periodização do treinamento de força em academias (2a ed.), Editora Manole.

Prestes, J., et al. (2013). Prescrição e periodização do treinamento de força em academias. (2a ed.), Manole, 2016.54 p. PRODANOV, Cleber Cristiano. Metodologia do Trabalho Científico: Métodos e Técnicas da Pesquisa e do Trabalho Acadêmico. Feevale.

Rodrigues, R. B. (2015). Alimentação saudável = saúde perfeita. (2a ed.), Clube dos Autores. S

Santarém, J. M. (2015). Musculação em todas as idades. Manole. 
Sharkey, B. (2015). Aptidão física ilustrada. Artmed.

Silva Filho, J. N. D., \& Ferreira, R. A. (2014). Treino de força: uma revisão sistemática sobre o volume de exercícios utilizados para emagrecimento. Colloquium Vitae. 1- 11 .

Souza, R. J. M. Treinamento de força rápida aplicado na preparação física de jovens tenistas. InterSciencePlace, 1, 37-56, 2015. http://interscienceplace.org/isp/index.php/isp/article/view/138/137.

Stábile, L., et al. (2017). Uma breve revisão: a utilização da suplementação de creatina no treinamento de força. Rev. Odontol. Araçatuba (Impr.), 38(1), 1418. https://pesquisa.bvsalud.org/portal/resource/pt/biblio-876028.

Tiggemann, C. L., Vanelli, C., Molinari, T., Kunrath, C. A., \& Dias, C. P. (2016). Efeito do alongamento prévio ao treinamento de força no desempenho de força máxima em homens jovens. Biomotriz.

Uchida, Marco Carlos. (2018). Manual de musculação. Phorte, 5.

Vieira, F. H. M., Barros, L.L., Martins, M. do C. de C., \& Cortez, A. C. L. (2015). O uso de suplementos alimentares, em praticantes de musculação: uma revisão de literatura Universidade Federal do Piauí, Centro de Ciências da Saúde.

Vieira, T. H. M. (2016). E colaboradores pode a suplementação da creatina melhorar o desempenho no exercício resistido? Revista Brasileira de Nutrição Esportiva. 10(55), 3-10.

Vieira, T. H. M., de Rezende, T. M., Gonçalves, L. M., Ribeiro, O. P. F., \& Silva Jr, A. J. (2016). Pode a suplementação da creatina melhorar o desempenho no exercício resistido? RBNE-Revista Brasileira de Nutrição Esportiva.

Williams, M. H., Kreider, R. B., \& Branch, J. D. (2017). Creatina. Manole, 212.

Word Anti-Doping Agency. (2015). Código Mundial Antidopagem. Quebec.

Zanelli, J. C. S., et al. (2015). Creatina e treinamento resistido: efeito na hidratação e massa corporal magra. Revista Brasileira de Medicina do Esporte, 21(1), 27-31https://www.scielo.br/scielo.php?pid=S1517-86922015000100027\&script=sci_arttext. 